\title{
On Some Freshwater Soft Algae from Bhulla Taal Lake Lansdowne, District Pauri Garhwal, Uttarakhand
}

Rakesh Kumar Dwivedi ${ }^{1 *}$

${ }^{1}$ Department of Botany, B.D. Govt. P.G. College, Jaiharikhal

Corresponding author Email id: dwivedirakeshji@gmail.com

Received: 4.10.2021; Revised: 12.12.2021; Accepted: 20.12.2021

(O) Society for Himalayan Action Research and Development

Abstract: Bhulla taal is a freshwater artificial lake, famous for tourist attraction in Lansdowne city of Pauri Garhwal district of Uttarakhand state, India. The lake is situated on Shivalik range of the Garhwal Himalaya. Morpho-taxonomic identification reveals total 26 freshwater soft algal taxa from the lake belonging to class Cyanophyceae and Chlorophyceae. Taxon Coenocystis subcylindrica Korshikov has been reported for the first time from the western Himalayan range.

Keywords: Freshwater algae, Pauri Garhwal, Uttarakhand

\section{Introduction}

Mapping of algal diversity in Himalayan region is a mammoth task. Morpho-taxonomic identification of freshwater soft algae is the part of biodiversity inventories. Sporadic reports have been made so far especially regarding freshwater soft algal taxa of Himalayan region. The Lansdowne city is situated at 1780 meter above msl, in Garhwal Himalaya; a part of Shivalik range of Western Himalaya. Bhulla taal, a famous tourist destination of the area, is an artificial small lake maintained by the army cantonment board of the Garhwal Rifles Regiment Centre. The lake has length 140.75 meter, width 62.81 meter and the area ca. $4004.98 \mathrm{~m}^{2}$. The lake is surrounded by the oak trees. It has inlet of water from northern end and the water goes out of the lake from its southern end and maintains the flow of water though out the year.

Sporadic reports on freshwater soft algae have been done form the Western Himalayan range. From Shivalik range of the Western Himalaya, fresh water soft algae have been reported by Dwivedi et al. (2006, 2008, 2008, 2009, 2014) mainly desmids, chlorococcalean and cyanophycean flora from Himachal Pradesh, from Kumaon region of the Uttarakhand, freshwater soft algae have been reported by Misra et al $(2007,2007)$ while that of from Garhwal Himalaya has been carried out by Habib (1998, 2001), Shukla et al. (2007, 2008), Misra et al. (2008) and Shukla et al. (2010).

\section{Material and method}

Freshwater algal samples were collected by random sampling method during summer seasons in May 2015 from the four different margins of the lake and in the middle of the lake through the boat. Epiphytic forms were collected by careful observations on the submerged plants. The samples were preserved with $4 \%$ formalin. Photomicrographs of the studied taxa were clicked by Nikon Labophot- II microscope in Phycology Laboratory of Lucknow University. 
Taxonomic enumeration, and dentification of the taxa have been done according to standard monographs of Smith (1933), Tiffany and Britton (1952), Desikachary (1959), Philipose (1967), Gonzalves (1981), Komarek \& Fott (1983), Prasad and Misra (1992) and authentic publications on freshwater algae of Prescott, G. W. (1961), da Silva and Felisberto, (2015) and Stastny (2010).

\section{Results and discussion}

\section{M. flos-aquae (Wittr.) Kirchner}

(Pl. 1, fig. 3)

Desikachary, T.V. (1959) (Pl. 17, fig. 11, pg. 94)

Cells 3-4 $\mu \mathrm{m}$ in diameter. Colonies roughly spherical, ellipsoidal, or somewhat elongate or often squarish in optical section, not clathrate, with indistinct colonial mucilage; cells spherical, with gas-vacuoles.

Collection no. \& date Lans/BT/01, 10/05/2015

\section{Choococcus limneticus Lemmermann}

(Pl. 1, fig. 1)

Desikachary, T.V. (1959) (Pl. 26, fig. 4-5, pg. 107)

Cells without sheath $10 \mu \mathrm{m}$ in dia., with sheath $14 \mu \mathrm{m}$ in dia, cells sub-spherical, sheath distinct, unlamellated, colourless, cell contents olive-green.

Collection no. \& date Lans/BT/03, 10/05/2015

\section{Merismopedia glauca (Ehr.) Naegeli}

(Pl. 1, fig. 2)

Desikachary, T.V. (1959) (Pl. 29, fig. 5, pg, 155)
Diameter of Cell, 3-4 $\mu \mathrm{m}$, dia. of colony 10 $\mu \mathrm{m}$, colony light blue green, almost rectangular with slightly sinuate-crenate margins cells; ovate or hemispherical, present in multiples of four, regularly arranged to form quadrangular colonies. Cell contents blue green homogenous, without granules but each cell have distinct centrally situated gas vacuole, cell wall smooth thick.

Collection no. \& date Lans/BT/01, 10/05/2015

\section{O. princeps Vaucher ex Gomont var. crassa Rao}

(Pl. 1, fig. 4)

Desikachary, T.V. (1959) (Pl. 39, fig. 11, pg. 206)

Trichome $45 \mu \mathrm{m}$ broad, $5 \mu \mathrm{m}$ long, bluegreen, more or less brownish, violet or reddish, mostly forming a thallus, mostly straight, not constricted at the cross-walls, blue-green to dirty green, slightly or briefly attenuated at the apices and bent, end cells flatly rounded, slightly capitate without or with slightly thickened membrane.

Collection no. \& date Lans/BT/01, 10/05/2015

\section{Oscillatoria peronata f. attenuata Skuja}

(Pl. 1, fig. 5)

Desikachary, T.V. (1959) (Pl. 41, fig. 14, pg. 205) Trichome $10 \mu \mathrm{m}$ broad, 3-4 um long.

Trichomes erect and flexuous, apices briefly attenuated and bent or curved, well constricted at the cross walls, $13-15 \mu \mathrm{m}$ broad, single or aggregated in floccose masses; cells finely granular, septa more or less granulated, end cell humilis depressed hemispherical, calyptra absent.

Collection no. \& date Lans/BT/02, 10/05/2015 


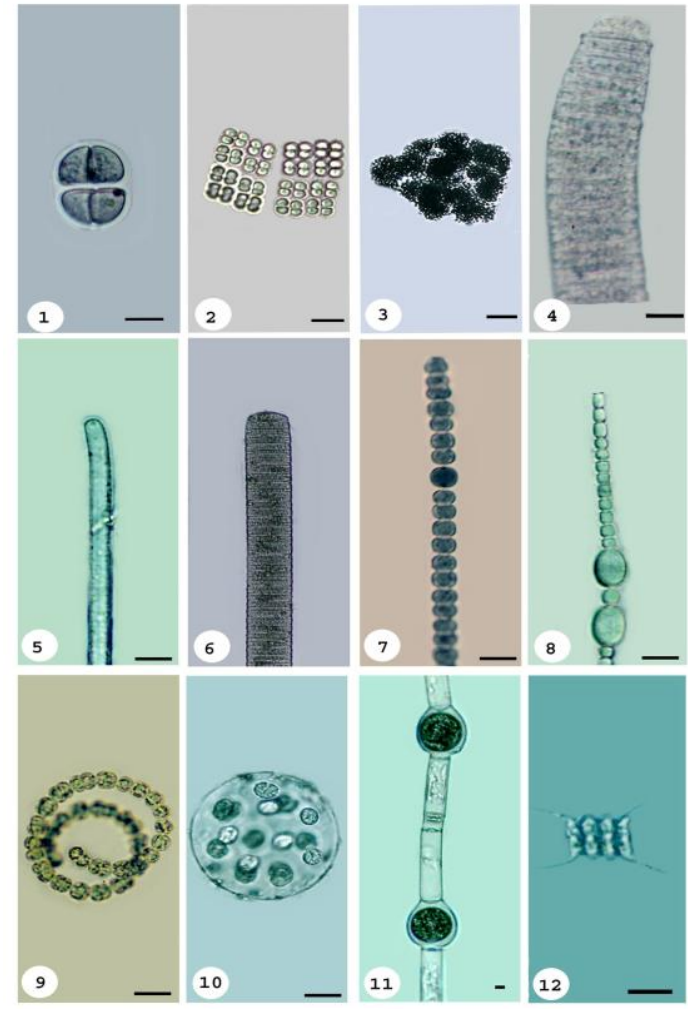

Fig Plate 1

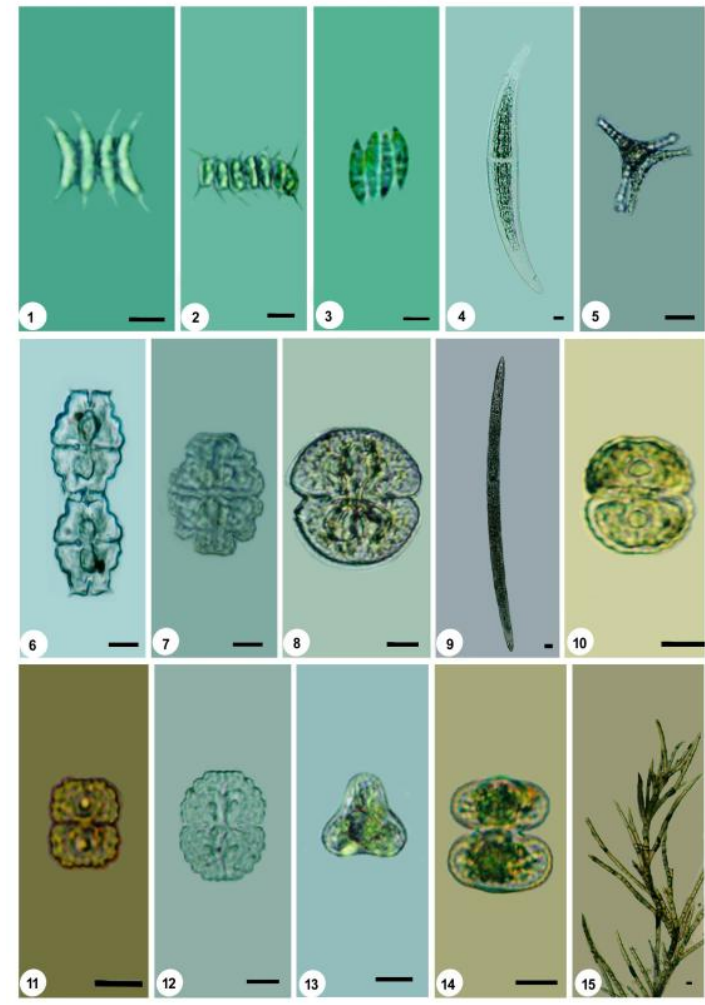

Fig Plate 2

Explanation of Plate -1 : 1. ChoococcuslimneticusLemmermann, 2. Merismopedia glauca (Ehr.) Naegeli, 3. Microcystis. flos-aquae (Wittr.) Kirchner, 4. Oscillatoria princeps Vaucher ex Gomont var. crassa Rao, 5. O.peronata f. attenuataSkuja, 6. O. sancta (Kuetz.) Gomont, 7. Anabaena macrosporaKlebahm, 8. A. iyengariiBharadwaja, 9. A. spiroidesKlebahn,10. CoenocystissubcylindricaKorshikov, 11. Oedogonium brevicingulatum var. brevicingulatumJao, 12. Scenedesmus tropicus Crow, All scales are equal to $10 \mu \mathrm{m}$

Explanation of Plate -2: 1. Scenedesmusacuminatus (Lagerheim) Chodat, 2. S. longusMeyen

3. S. arcuatus var. capitatus G.M. Smith, 4. Closterium moniliferum (Bory) Ehrenberg,

5. Staurastrumneglectum G.S. West, 6. Euastrumsinuosum var. reductum West \& West

7. E subornatum West \& West var. brazilienseBorge, 8. Cosmariumobsoletum (Hantzsch) Reinsch, 9. Closterium brauniiReinsch, 10. Cosmariumawadhense Prasad \& Mehrotra, 11. C. medioretusumCoesel, 12. C. ornatum Eichler \&Gutwinski, 13\&14. Staurastrumlunatum Ralf, 15. Stigeoclonium tenue (Agardh) Kuetzing, All scales are equal to $10 \mu \mathrm{m}$ except fig. $4,9 \& 15$ which are equal to $50 \mu \mathrm{m}$

\section{O. sancta (Kuetz.) Gomont}

(Pl. 1, fig. 6)

Desikachary, T.V. (1959) (Pl. 42, fig. 10, Pg. 203)

Trichome $2.5 \mu \mathrm{m}$ long, $11 \mu \mathrm{m}$ broad, thallus dark green, trichome slightly bent, distinctly Collection no. \& date Lans/BT/02, 10/05/2015

\section{Anabaena macrospora Klebahm}

(Pl. 1, fig. 7)
Prescott, G. W. (1961) (Fig. 4-6, pl. 117, pg. 517)

Cells $7 \mu \mathrm{m}$ in width, heterocyst $7 \mu \mathrm{m}$ in width. Trichomes planktonic, straight or flexuous, solitary; cells globose or somewhat ellipsoid, 5-6.5 $\mu \mathrm{m}$ in dia., with abundant pseudovacuoles; heterocyst spherical about $6 \mu \mathrm{m}$ in diameter, spores not adjoining the heterocyst.

Collection no. \& date Lans/BT/01, 10/05/2015

\section{A. iyengarii Bharadwaja}


(Pl. 1, fig. 8)

Desikachary, T.V. (1959) (Pl. 76, fig. 1, pg. 406)

Cells 5-6 $\mu \mathrm{m}$ in width, heterocyst $7 \mu \mathrm{m}$ in width, spores $8 \mu \mathrm{m}$ in width and $10-11 \mu \mathrm{m}$ in length, trichome single, cell barrel shaped, slightly longer than the broad, end cells with rounded apex, heterocyst spherical, spores ellipsoidal on either side of the heterocyst. Epispore thick and smooth.

Collection no. \& date Lans/BT/04, 10/05/2015

\section{A. spiroides Klebahn}

(Pl. 1, fig. 9)

Desikachary, T.V. (1959) (Pl. 71, fig. 9, pg. 395)

Cells 7-8 $\mu \mathrm{m}$ broad

Trichome free floating, single, regularly spirally coiled, spirals $45-55 \mu \mathrm{m}$ broad, cells spherical with distinct gas vacuoles.

Collection no. \& date Lans/BT/01, 10/05/2015

\section{Coenocystis subcylindrica Korshikov}

(Pl. 1. Fig. 10)

Komárek and Fott (1983) (Tafel 124, fig 1,pg. 411)

Colonies are spherical, oval, slightly irregular or several sub-colonies, arranged parallel in 1 or 2 planes, slightly irregularly, with a distance from each other. Cells are widely ovoid or sometimes slightly asymmetrical shape. Chloroplast is parietal, massive, without pyrenoid. Cells are 5-7 $\mu \mathrm{m}$ in length and 3-5 $\mu \mathrm{m}$ in width.

Collection no. \& date Lans/BT/01, 10/05/2015

11. Oedogonium brevicingulatum var. brevicingulatum Jao

(Pl. 1 fig. 11)
Gonzolves (1982) (Fig. 9.22A, pg.159)

Macrandrous, homothallic, vegetative cells cylindric $32 \mu \mathrm{m}$ broad, basal cell short, oogonium single, obovoid, $51 \mu \mathrm{m}$ in diameter, poriferous, pore superior, oospore globose not filling the oogonium, spore wall smooth Collection no. \& date Lans/BT/02, 10/05/2015

\section{Scenedesmus tropicus Crow}

(Pl. 1 fig. 12)

Philipose (1967) (Fig. 185, pg. 279)

Colony 4-celled, subquadrate, cells more or less biconvex in middle attenuated towards apex with inflated poles. Poles of terminal cells provided with long recurved spine. Chloroplast parietal with a large single pyrenoid. Cells $15 \mu \mathrm{m}$ long, 4-6 $\mu \mathrm{m}$ broad, spines $12 \mu \mathrm{m}$ long.

Collection no. \& date Lans/BT/03, 10/05/2015

\section{S. acuminatus (Lagerheim) Chodat}

(Pl. 2 fig. 1)

Philipose, M.T. (1967) (Fig 161a, pg. 251)

Cell $20 \mu \mathrm{m}$ long, $4 \mu \mathrm{m}$ broad. Colonies curved and of 4-8 fusiform cells with sharp pointed ends. All the cells in a colony lunate or the interior cells forming a flat plate.

Collection no. \& date Lans/BT/04, 10/05/2015

\section{S. longus Meyen}

(Pl. 2 fig. 2)

Philipose, M.T. (1967) (Fig 180a, pg. 273)

Colony flat, eight cells arranged in a single linear siries, cells oblong cylindrical with rounded poles. Poles with 2 spines at each end. Cell 8-10 $\mu \mathrm{m}$ long, 4-5 $\mu \mathrm{m}$ broad, spines 8-12 $\mu \mathrm{m}$ long.

Collection no. \& date Lans/BT/04, 10/05/2015

15. S. arcuatus var. capitatus G.M. Smith 
(P1. 2. Fig. 3)

Philipose, M.T. (1967) (Fig 166k, pg. 257)

Colony curved, 4 celled, in sublinear series.

Cells slightly curved with one side convex and other slightly concave. End of cells stumpy with nodular thickenings. Cells $5 \mu \mathrm{m}$ broad, 12-15 $\mu \mathrm{m}$ long.

Collection no. \& date Lans/BT/01, 10/05/2015

16. Closterium moniliferum (Bory)

\section{Ehrenberg}

(Pl. 2. Fig. 4)

Tiffany L.H. \& Britton, M.E. (1992) (Pl. 52, fig. 549, pg. 172)

Cell $305 \mu \mathrm{m}$ long, $46 \mu \mathrm{m}$ broad, and apex 6 $\mu \mathrm{m}$. Cell stout, moderately curved, inner margin inflated in the middle, cell uniformly narrowed with rounded apex. Cell wall smooth, chloroplast with 6 ridges with pyrenoids and vacuoles.

Collection no. \& date Lans/BT/03, 10/05/2015

\section{Staurastrum neglectum G.S. West}

(Pl. 2. Fig. 5)

Coesel, P.F.M. \& Meesters, K.J. 2013. (Pl. 95:

1-3, pg. 124)

Body of semi-cell in apical view is triradiate, cell $20 \mu \mathrm{m}$ in length twisted at isthmus, processes ornamented, end of the processes tipped with minute spines.

Collection no. \& date Lans/BT/03, 10/05/2015

18. Euastrum sinuosum var. reductum West

\section{\& West}

(Pl. 2, fig. 6)

Prasad \& Misra 1992. (Pl 19, fig. 2, pg. 135)

Cells small, 1.7 times longer than broad, deeply constricted, sinus narrowly linear with dilated extremity, lateral lobes bilobulate and less prominent, polar lobe quadrate oblong with deep median incision, punctation on cell wall not seen. Cell $40 \mu \mathrm{m}$, in length, width 21 $\mu \mathrm{m}$, isthmus $5 \mu \mathrm{m}$.

Collection no. \& date Lans/BT/03, 10/05/2015

19. E subornatum West \& West var. braziliense Borge

(P1.2 fig 7)

da Silva F.K.L. \& Felisberto, S.A. (2015) (fig. $2 \mathrm{j})$

Cell slightly longer than wide, 40 x $30 \mu \mathrm{m}$ isthmus $8 \mu \mathrm{m}$ with median constriction, central linear sine, fully open, opening to the isthmus; trapezoidal semicell, lateral lobes rounded; slightly truncated polar lobe; semicell with tumescence in the midline of the lateral lobes formed by a single ring of granules; cell wall ornate with granules.

Collection no. \& date Lans/BT/04, 10/05/2015

20. Cosmarium obsoletum (Hantzsch)

\section{Reinsch}

(P1. 2. Fig 8)

Prasad \& Misra 1992. (Pl. 22. Fig. 16 pg. 170)

Cell $58 \mu \mathrm{m}$ long, $65 \mu \mathrm{m}$ broad, and isthmus 20 $\mu \mathrm{m}$, transversely elliptic, slightly broader than the long, deeply constricted, sinus narrow with dilated apex and slightly open outwards, cell wall punctate, chloroplast axile with two pyrenoids in each semicells.

Collection no. \& date Lans/BT/04, 10/05/2015

\section{Closterium braunii Reinsch}

(Pl. 2 fig. 9)

Tiffany \& Britton (1952) (Pl. 51, fig. 541, pg. 176) 
Cell $720 \mu \mathrm{m}$ long, $40 \mu \mathrm{m}$ broad, apex $8 \mu \mathrm{m}$. wall yellowish and very finely striate, becoming brown at poles which are punctate.

Collection no. \& date Lans/BT/03, 10/05/2015

\section{Cosmarium awadhense Prasad \&}

\section{Mehrotra}

(Pl. 2, fig. 10)

Prasad \& Misra (1992) (Pl. 21, fig. 27, pg. 153)

Cell $26 \mu \mathrm{m}$ long, $18 \mu \mathrm{m}$ broad, isthmus 14 $\mu \mathrm{m}$. cell small, slightly longer than broad, sinus narrowly linear toward apex and slightly open, semicells sub-semicircular, apex truncate with more or less straight margin, each semicell with one large chloroplast and one pyrenoid.

Collection no. \& date Lans/BT/04, 10/05/2015

\section{C. medioretusum Coesel}

(Pl. 2. Fig. 11)

Stastny (2010) (fig. 166)

Cell small, $18 \mu \mathrm{m}$ long, $12 \mu \mathrm{m}$ broad, isthmus $6 \mu \mathrm{m}$, apex of semicells flattened, margin crenated, chloroplast with one pyrenoid.

Collection no. \& date Lans/BT/04, 10/05/2015

\section{C. ornatum Eichler \& Gutwinski}

(Pl. 2. Fig. 12)

Prescott et.al. (1981) (Pl. 256, Fig. 6, pg. 239)

Cell medium sized, slightly longer than the borad, length $34 \mu \mathrm{m}$, width $28 \mu \mathrm{m}$, isthmus 15 $\mu \mathrm{m}$, semicell with narrow sinus opening, chloroplast with two pyrenoids in each semicell.

Collection no. \& date Lans/BT/02, 10/05/2015

\section{5 \& 26. Staurastrum lunatum Ralf}

(Pl. 2 fig. 13, 14)

Prescott (1940) (P1 II, Fig. 20-21, pg. 9)
Length $26 \mu \mathrm{m}$, width with processes $29 \mu \mathrm{m}$, isthmus $10 \mu \mathrm{m}$

Collection no. \& date Lans/BT/02, 10/05/2015

27. Stigeoclonium tenue (Agardh) Kuetzing (Pl. 2 fig. 15)

Tiffany \& Britton (1952) (Pl 10, fig. 70, pg. 34)

Cell 7-10 $\mu \mathrm{m}$ long, and 5-8 $\mu \mathrm{m}$ broad, branches more often opposite, some always solitary, tuft bright green.

Collection no. \& date Lans/BT/02, 10/05/2015

Out of total 26 taxa reported from the Bhulla lake, the taxon Coenocystis subcylindrica Korshikov has been reported for the first time from the western Himalayan range.

\section{Acknowledgement}

Author is thankful to the Head, Department of Botany, University of Lucknow for providing the facilities of microphotography and literature.

\section{References}

Brook A.J (1992). Desmids of the Staurastrum tetracerum-group from a eutrophic lake in mid-wales. Brit. Phycol. J.17. 259274

da Silva F.K.L. \& Felisberto, S.A. (2015) Euastrum and Micrasterias (family Desmidiaceae) in lentic tropical ecosystem, Brazil. Biota Neotropica. 15(1): 1-12.

Desikachary TV (1959). Cyanophyta. I.C.A.R. Monograph on algae, New Delhi. 686p. Dwivedi RK, Misra PK, Shukla CP, Tripathi SK (2008). On some Desmids from 
Southern Himachal Pradesh of Western

Himalaya and its foothills.

Phytotaxonomy. 8: pg 83-86.

Dwivedi RK, Shukla SK. Shukla CP, Misra

PK, Seth MK (2008). Cyanophycean algal flora of Southern Himachal Pradesh, India. Ecoprint. 15: 29-36 pp.

Dwivedi RK, Shukla CP, Misra P.K., Shukla SK., Seth M.K. (2009) On some freshwater desmids from Southern Himachal Pradesh of Indo-Western Himalaya. Feddes Repertorium. 123: 34, pp. 236-249.

Dwivedi R.K. \& Misra P.K. (2013). On the occurrence of three new taxa of freshwater algae Lagerheimia Chodat from Indo-western Himalaya. Phykos. 43(1). 46-50.

Dwivedi R.K. \& Misra P.K. (2014). On some freshwater algae of district Una, Himachal Pradesh, India. Phytotaxonomy. 14. 103-106.

Gonzalves, E.A. (1982) Oedogoniales, I.C.A.R. monograph on algae. New Delhi. 757p.

Habib, I. Ghildiyal, J.C., Negi, C. and Chaturvedi, U.K. (1998). A systematic account of

Chlorococcales from Kotdwar, Garhwal. Phykos 31 (1-2): 125-129.

Habib I (2001). A preliminary study on certain algal flora from the foothills zone of Garhwal Himalaya. J. of Econ. and Taxon. Bot. 25(2): 317-322.

Komárek J, Fott B (1983) Das Phytoplankton des Süsswasers. Stuttgart. Germany. 1-
1044.Misra, P.K., Rai, A.K., Chauhan, R.S. and Shukla, S.K. (2007a). Algal flora of Naukuchia Tal, Nainital district, Uttaranchal state. J. of Ind. Bot. Soc. 86(1\&2): 71-77.

Misra PK, Rai AK, Shukla SK (2007). Some fresh water desmids from Kumaon Himalaya. J. of App. Biosciences, 33(1): 53-58.

Misra PK, Misra ., Shukla, M., and Prakash, J. (2008) Some desmids from Garhwal region of Uttarakhand, India. Algae. 23(3): 177-186.

Shukla SK, Shukla CP, Misra PK (2008). Desmids (Chlorophyceae, Conjugales, Desmidiaceae) from foothills of Western Himalaya, India. Algae, 23(1): $1-14$.

Shukla Chandra Prakash, Singh DB, Shukla SK, Misra PK (2010). Studies on Cyanophycean Algae of Garhwal Region of Uttarakhand State. Res. Dimension. 1; 12-17.

Philipose, M.T. (1967). Chlorococcales, I.C.A.R. Monograph on algae. New Delhi. 365p.

Tiffany LH, Britton ME (1952). The algae of Illinois. Hafner publishing Co., New York. 407p.

Prasad B.N. and Mishra P.K. (1992) Monograph on fresh water algal flora of Andman and Nicobar islands. Vol.2. Bisen Singh, Mahendra Pal Singh, Dehradun, 284pp. 
Smith GM (1933). The fresh water algae of the United States, McGraw-Hill Book Company Inc., New York, 716p.

Statsny J (2010). Desmids (Conjugatophyceae, Viridiplantae) from the Czech Republic; new and rare taxa, distribution, ecology.

Fottea. 10(1), 1-74. 